\title{
Tendência temporal da tuberculose drogarresistente (TBDR) e dos tipos de resistência no estado de Pernambuco, Brasil
}

\section{Temporal trend of Drug-Resistant Tuberculosis (DRTB) and resistance types in the state of Pernambuco, Brazil.}

Ariane Cristina Bezerra Silva Martins', Ulisses Montarroyos², Amanda Queiroz', Jadson Mendonça Galindo ${ }^{3}$, Michelle Christiane da Silva Rabello ${ }^{4}$ (D), Haiana Charifker Schindler ${ }^{4}$

'Secretaria de Saúde da Prefeitura da Cidade do Recife - Recife (PE), Brasil.

${ }^{2}$ Instituto de Ciências Biológicas, Universidade de Pernambuco - Recife (PE), Brasil.

${ }^{3}$ Secretaria de Saúde do Estado de Pernambuco - Recife (PE), Brasil.

${ }^{4}$ Departamento de Imunologia, Instituto Aggeu Magalhães da Fundação Oswaldo Cruz - Recife (PE), Brasil.

Como citar: Martins ACBS, Montarroyos U, Queiroz A, Galindo JM, Rabello MCS, Schindler HC. Tendência temporal da tuberculose drogarresistente (TBDR) e dos tipos de resistência no estado de Pernambuco, Brasil. Cad Saúde Colet, 2021; 29(3):399-410. https://doi.org/10.1590/1414-462X202129030177.

\section{Resumo}

Introdução: A tuberculose drogarresistente (TBDR) é um dos grandes desafios para a saúde pública. Objetivo: Este estudo analisou a tendência temporal da TBDR e a razão da proporção dos tipos de resistência no estado de Pernambuco, no período de 2002 a 2014. Método: Trata-se de um estudo observacional do tipo tendência temporal de série histórica utilizando dados do Sistema de Informação de Tratamentos Especiais da Tuberculose e análise pelo modelo autorregressivo de médias móveis de ordem 2. Resultados: Foram notificados 298 novos casos de TBDR, com incidência variando entre 0,06 e 0,71/100 mil habitantes. Pelo menos 1 caso foi notificado em 40 dos 185 municípios do estado. A tendência temporal da TBDR e da proporção entre os tipos de resistência não apresentou comportamento linear. Nos últimos cinco anos, houve uma tendência ascendente, concentrando $71,1 \%$ dos casos da série histórica. Em 2014, foi observado um crescimento expressivo tanto da TBDR primária como da secundária, quando comparado ao ano de 2002. Conclusão: Apesar da baixa incidência e de a tendência da TBDR não ter comportamento linear, o aumento de casos nos últimos anos da série histórica pode ser considerado um sinal de alerta para os programas de controle da doença em Pernambuco.

Palavras-chave: tuberculose; tuberculose resistente a múltiplos medicamentos; epidemiologia; estudos de séries temporais.

\begin{abstract}
Background: Drug-Resistant Tuberculosis (DRTB) is one of the most relevant challenge to public health. Objective: This study analyzed the temporal trend of the DRTB and resistance types in the state of Pernambuco, from 2002 to 2014. Method: This was an observational study of the temporal trend type of a historical series using data from the Special Treatment Information System of Tuberculosis (SITE-TB) and also analysis by the autoregressive moving average models of order 2. Results: A total of 298 new DRTB cases were reported, ranging from 0.06 to 0.71 per 100,000 inhabitants. At least one case was reported in 40 of the 185 municipalities in the state. The temporal trend of DRTB and the proportion between resistance types did not present a linear behavior. In the last 5 years, there has been an upward trend,
\end{abstract}

Este é um artigo publicado em acesso aberto (Open Access) sob a licença Creative Commons Attribution, que permite uso, distribuição e reprodução em qualquer meio, sem restrições desde que o trabalho original seja corretamente citado.

\footnotetext{
Trabalho realizado no Instituto Aggeu Magalhães/Fiocruz - Recife (PE), Brasil.

Correspondência: Michelle Christiane da Silva Rabello. E-mail: michelle.rabello@cpqam.fiocruz.br

Fonte de financiamento: nenhuma.

Conflito de interesses: nada a declarar.

Recebido em: Maio 14, 2019. Aprovado em: Abr. 09, 2020
} 
concentrating $71.1 \%$ of the historical series. In 2014, there was an expressive growth of both primary and secondary DRTB, when compared to the year 2002. Conclusion: Despite the low incidence and tendency of DRTB not to be linear, the increase in cases in the last years of the historical series can be considered as a warning sign for the disease control programs of Pernambuco.

Keywords: tuberculosis; multidrug-resistant tuberculosis; epidemiology; time series studies.

\section{INTRODUÇÃO}

A tuberculose (TB) é uma das doenças transmissíveis com maior destaque no mundo. Em 2016, 6,3 milhões de novos casos da doença foram notificados, dos quais cerca de 480 mil seriam de tuberculose drogarresistente (TBDR) ${ }^{1}$ e mais da metade deles estariam na Índia, China e Rússia² ${ }^{2}$ Em 2014, ocorreram, em média, 1,5 milhão de mortes relacionadas à TB³ .

A TBDR tornou-se uma grande ameaça para o controle da doença no mundo. As taxas de cura desses casos são mais baixas ${ }^{3-5}$ se comparadas às taxas de cura dos casos de TB sensível às drogas de primeira linha recomendadas pelo Ministério da Saúde do Brasil ${ }^{6}$. A TBDR é caracterizada quando o microrganismo Mycobacterium tuberculosis apresenta resistência a pelo menos uma das drogas usadas no tratamento da doença. No Brasil, a resistência a um fármaco do tratamento para TB é definida como monorresistência. A polirresistência ocorre quando há resistência a dois ou mais fármacos de primeira linha usados no tratamento da doença, exceto se a resistência estiver associada às drogas rifampicina $(R)$ e isoniazida $(H)$, que, neste caso, é considerada como tuberculose multirresistente (TB-MDR).

O problema da resistência às drogas se agravou com o surgimento das formas mais difíceis de tratar, como a TB-MDR e a forma extensivamente resistente (TB-XDR), extensively drug resistant tuberculosis-9 ${ }^{7-}$ A TB-XDR, além das resistências presentes na TB-MDR, apresenta resistência a pelo menos uma droga em uma das duas classes de drogas de segunda linha usadas para o tratamento de TB-MDR: fluoroquinolonas e aminoglicosídeos injetáveis ${ }^{6}$.

Diversos estudos mostram que o percentual de casos de TBDR vem crescendo ao longo dos anos em diversos países, incluindo o Brasil, com maior acometimento de indivíduos do sexo masculino, na faixa etária economicamente ativa e com padrão de resistência a mais de uma droga ${ }^{10,11}$.

No Brasil, em 2014, foram notificados 374 casos de multirresistência e 56 de resistência extensiva no Sistema de Informação de Tratamentos Especiais da Tuberculose (SITETB), que é um sistema on-line para notificação dos casos diagnosticados que necessitam de tratamentos especiais, entre os quais os casos de TBDR ${ }^{12,13}$. Dos estados brasileiros, Pernambuco se destaca por ser o terceiro em maior taxa de coeficiente de incidência de TB e o segundo em maior risco para o óbito pela doença ${ }^{14}$. Em 2012, ocupou o quarto lugar em números de casos notificados de TBDR ${ }^{15}$.

Assim como os inquéritos epidemiológicos nacionais de TB, estudos epidemiológicos de série histórica constituem uma importante ferramenta auxiliar para o monitoramento dos programas de controle da TBDR em várias regiões brasileiras, pois permitem identificar os locais e o período em que o problema se apresenta com maior gravidade ${ }^{16}$. Neste contexto, este estudo obteve o perfil da TBDR no estado de Pernambuco e analisou a tendência temporal da incidência e da proporção dos casos novos de TBDR primária e secundária notificados no período de 2002 a 2014.

\section{MÉTODO}

Trata-se de um estudo observacional de série histórica com dados de fonte secundária do perfil epidemiológico e da tendência temporal da incidência da TBDR e da razão entre os tipos de casos de resistência em Pernambuco, um dos estados-membros da região Nordeste do Brasil. O estudo incluiu o total de casos novos de TBDR notificados no SITETB no período de 2002 a 2014.

Pernambuco é constituído por 184 municípios e possui 12 Gerências Regionais de Saúde (GERES), organizadas da seguinte forma: I - Recife; II - Limoeiro; III - Palmares; IV - Caruaru; V Garanhuns; VI - Arcoverde; VII - Salgueiro; VIII - Petrolina; IX - Ouricuri; X - Afogados da Ingazeira; 
XI - Serra Talhada; e XII - Goiana. Cada uma dessas unidades administrativas da Secretaria Estadual de Saúde é responsável por uma parte dos municípios do estado, atuando de forma mais localizada na atenção básica de saúde e na reestruturação da rede hospitalar ${ }^{17}$.

Neste estudo, a TBDR primária foi definida como aqueles casos em que o paciente não apresentava história ou antecedentes de tratamento anti-TB, e a TBDR secundária, como aqueles que já possuíam relato de uso de drogas anti-TB por mais de 30 dias. A frequência da totalidade dos dados sociodemográficos e clínico-epidemiológicos - sexo, idade, padrão de resistência, forma clínica da doença, tratamento diretamente observado (TDO), desfecho do caso, município e GERES de residência do caso - dos pacientes com TBDR incluídos na série histórica foi extraída do SITETB.

Para o cálculo e a análise da incidência, a TBDR foi descrita por ano de diagnóstico dos casos notificados, e a população geral foi considerada como a população exposta. Portanto, no numerador, foram considerados todos os casos novos notificados de TBDR (primária e secundária), e no denominador, a população geral de Pernambuco por ano do período estudado. O levantamento da proporção entre os casos de resistência primária $(A)$ e secundária (B) foi realizado por meio do cálculo da razão (A/B), por ano, do total de casos de TBDR.

O modelo de séries temporais que melhor se adequou ao cálculo e à análise do comportamento da tendência da incidência da TBDR e da razão entre os tipos de casos de resistência foi o modelo autorregressivo de médias móveis de ordem 2. Por não apresentar um componente cíclico ou sazonal, foi aplicada uma análise utilizando a abordagem Box-Jenkins para ajustar o modelo autorregressivo de médias móveis de ordem $2^{18}$. A análise estatística para a estimativa do modelo e da série temporal foi realizada usando o software $R$, versão 3.3.3.

O estudo teve anuência da Secretaria de Saúde do Estado de Pernambuco e aprovação pelo Comitê de Ética em Pesquisa do Instituto Aggeu Magalhães/Fiocruz (Parecer: CAAE: 53416016.5.0000.5190). Por se tratar de dados de fonte secundária, não foi necessária a aplicação do Termo de Consentimento Livre e Esclarecido.

\section{RESULTADOS}

Em Pernambuco, foram notificados 298 novos casos de TBDR no SITETB entre o período de 2002 a 2014. De acordo com o padrão de resistência, a TB- MDR foi diagnosticada na maioria dos casos $(79,2 \% ; n=236)$. A TBDR acometeu $69 \%(n=207)$ do sexo masculino, e a faixa etária predominante foi entre 25 e 49 anos $(63,8 \% ; n=190)$. Dos 298 pacientes notificados com TBDR, $98,3 \%(n=293)$ apresentaram a forma pulmonar (Tabela 1).

Em relação ao tratamento, a maioria $(61,7 \% ; n=124$ casos) não teve acompanhamento domiciliar, ou seja, TDO, e 41,6\% ( $n=124)$ apresentaram desfecho não favorável (abandono, óbito, falência, mudança de diagnóstico e mudança de esquema). $\mathrm{O}$ abandono do tratamento ( $\mathrm{n}$ $=48$ ) e o óbito porTBDR $(n=44)$ foram os desfechos não favoráveis mais frequentes (Tabela 1).

A distribuição da TBDR por município e GERES mostra que, no período estudado, 40 dos 185 municípios do estado tiveram ao menos 1 caso novo de TBDR registrado. Desses municípios, 13 notificaram casos de TBDR primária. No entanto, em 3 municípios (Itapissuma, Gameleira e Ribeirão) foram observados somente casos primários, não apresentando TBDR secundária (Tabela 2).

Em relação à distribuição por GERES, a I GERES apresentou a maior carga da doença, com $86,8 \%(n=257)$ dos casos de TBDR. As maiores frequências foram identificadas em municípios dessa mesma região. Em Recife, foram encontrados $40 \%(n=118)$ dos casos de TBDR, seguidos dos municípios de Jaboatão dos Guararapes, Cabo de Santo Agostinho e Olinda, com 14,2\% $(n=42), 11,8 \%(n=35)$ e $4,5 \%(n=13)$, respectivamente. Quanto à taxa de incidência da TBDR por município, Ilha de Itamaracá (32,0/100 mil), Cabo de Santo Agostinho (18,9/100 mil habitantes), Verdejante (10,9/100 mil habitantes), Moreno (10,6/100 mil habitantes) e Buenos Aires $(8,0 / 100$ mil habitantes) foram os municípios com as maiores chances de ocorrência da TBDR (Tabela 2).

A taxa de incidência anual da TBDR no período da série histórica estudada variou de 0,06 a $0,71 / 100$ mil habitantes. No último quinquênio, foram identificadas as maiores 
Tabela 1. Caracterização sociodemográfica e clínico-epidemiológica dos casos novos de tuberculose drogarresistente (TBDR) notificados, Pernambuco, 2002-2014

\begin{tabular}{|c|c|c|c|}
\hline \multirow{3}{*}{ Variáveis } & \multicolumn{3}{|c|}{ Tuberculose drogarresistente } \\
\hline & Primária & Secundária & Total \\
\hline & $\mathbf{N}(\%)$ & $\mathbf{N}(\%)$ & $\mathbf{N}(\%)$ \\
\hline \multicolumn{4}{|l|}{ Padrão de resistência } \\
\hline Monorresistência & $14(24,1)$ & $31(12,9)$ & $45(15,1)$ \\
\hline Polirresistência & $4(6,9)$ & $7(2,9)$ & $11(3,7)$ \\
\hline Multirresistência & $38(65,5)$ & $198(82,5)$ & $236(79,2)$ \\
\hline Resistência extensiva & $2(3,5)$ & $4(1,7)$ & $6(2,0)$ \\
\hline \multicolumn{4}{|l|}{ Sexo } \\
\hline Masculino & $37(63,8)$ & $170(70,8)$ & $207(69,5)$ \\
\hline Feminino & $21(36,2)$ & $70(29,2)$ & $91(30,5)$ \\
\hline \multicolumn{4}{|l|}{ Faixa etária } \\
\hline 0 a 24 anos & $8(13,8)$ & $28(11,7)$ & $36(12,0)$ \\
\hline 25 a 49 anos & $33(56,9)$ & $157(65,4)$ & $190(63,8)$ \\
\hline 50 a 59 anos & $13(22,4)$ & $37(15,4)$ & $50(16,8)$ \\
\hline 60 ou + anos & $4(6,9)$ & $18(7,5)$ & $22(7,4)$ \\
\hline \multicolumn{4}{|l|}{ Forma clínica da TBDR } \\
\hline Pulmonar & $57(98,3)$ & $236(98,3)$ & $293(98,3)$ \\
\hline Mista & $0(0)$ & $4(1,7)$ & $4(1,4)$ \\
\hline Extrapulmonar & $1(1,7)$ & $0(0)$ & $1(0,3)$ \\
\hline \multicolumn{4}{|l|}{ Tratamento diretamente observado (TDO) } \\
\hline Sim & $23(39,7)$ & $91(37,9)$ & $114(38,3)$ \\
\hline Não & $35(60,3)$ & $149(62,1)$ & $184(61,7)$ \\
\hline \multicolumn{4}{|l|}{ Desfecho do caso } \\
\hline $\begin{array}{l}\text { Favorável (cura, tratamento completo, em } \\
\text { tratamento) }\end{array}$ & $36(62,1)$ & $138(57,5)$ & $174(58,4)$ \\
\hline Curado & $11(30,5)$ & $37(26,8)$ & $48(27,6)$ \\
\hline Tratamento completo & $24(66,7)$ & $93(67,4)$ & $117(67,2)$ \\
\hline Em tratamento & $1(2,8)$ & $8(5,8)$ & $9(5,2)$ \\
\hline $\begin{array}{l}\text { Não favorável (abandono, óbito, falência, } \\
\text { mudança de esquema, }\end{array}$ & $22(37,9)$ & $102(42,5)$ & $124(41,6)$ \\
\hline \multicolumn{4}{|l|}{ mudança de diagnóstico) } \\
\hline Abandono & $8(36,4)$ & $40(39,2)$ & $48(38,7)$ \\
\hline Óbito & $7(31,8)$ & $37(36,3)$ & $44(35,5)$ \\
\hline Falência & $7(31,8)$ & $23(22,5)$ & $30(24,2)$ \\
\hline Mudança de diagnóstico & $0(0)$ & $1(1)$ & $1(0,8)$ \\
\hline Mudança de esquema & $0(0)$ & $1(1)$ & $1(0,8)$ \\
\hline Total & $58(19,5)$ & $240(80,5)$ & 298 \\
\hline
\end{tabular}

taxas de incidência, com média de 0,47/100 mil habitantes, atingindo pico máximo $(0,71 / 100$ mil habitantes) no ano de 2012, no qual foram registrados 63 casos de TBDR (Figura 1). 
Tabela 2. Distribuição dos casos novos e taxa de incidência de tuberculose drogarresistente (TBDR) notificados por município e região de saúde, Pernambuco, 2002-2014

\begin{tabular}{|c|c|c|c|c|c|}
\hline \multirow[b]{2}{*}{$\begin{array}{l}\text { Região } \\
\text { de saúde/ } \\
\text { município }\end{array}$} & \multicolumn{5}{|c|}{ Tuberculose drogarresistente } \\
\hline & Secundária & Primária & Total & $\%$ & $\begin{array}{c}\text { Taxa de } \\
\text { incidência } \\
\text { (Total de } \\
\text { casos/ } \\
\text { habitantes } \\
\text { Censo } 2010 \mathrm{x} \\
100 \text { mil) }\end{array}$ \\
\hline $\begin{array}{l}\text { I Região de } \\
\text { saúde }\end{array}$ & 205 & 52 & 257 & 86,8 & ---- \\
\hline Abreu e Lima & 2 & 0 & 2 & 0,7 & 2,1 \\
\hline $\begin{array}{l}\text { Cabo de Santo } \\
\text { Agostinho }\end{array}$ & 30 & 5 & 35 & 11,8 & 18,9 \\
\hline Camaragibe & 6 & 0 & 6 & 2 & 4,2 \\
\hline Igarassu & 5 & 3 & 8 & 2,7 & 7,8 \\
\hline $\begin{array}{l}\text { Ilha de } \\
\text { Itamaracá }\end{array}$ & 7 & 0 & 7 & 2,3 & 32,0 \\
\hline Ipojuca & 2 & 1 & 3 & 1 & 3,7 \\
\hline Itapissuma & 0 & 1 & 1 & 0,3 & 4,2 \\
\hline $\begin{array}{l}\text { Jaboatão dos } \\
\text { Guararapes }\end{array}$ & 31 & 11 & 42 & 14,2 & 6,5 \\
\hline Moreno & 5 & 1 & 6 & 2 & 10,6 \\
\hline Olinda & 11 & 2 & 13 & 4,5 & 3,4 \\
\hline Paulista & 6 & 2 & 8 & 2,7 & 2,7 \\
\hline Recife & 92 & 26 & 118 & 40 & 7,7 \\
\hline $\begin{array}{l}\text { São Lourenço } \\
\text { da Mata }\end{array}$ & 1 & 0 & 1 & 0,3 & 1,0 \\
\hline $\begin{array}{l}\text { Vitória de } \\
\text { Santo Antão }\end{array}$ & 7 & 0 & 7 & 2,3 & 5,4 \\
\hline $\begin{array}{l}\text { II Região de } \\
\text { saúde }\end{array}$ & 7 & 0 & 7 & 2,2 & --- \\
\hline Buenos Aires & 1 & 0 & 1 & 0,3 & 8,0 \\
\hline Carpina & 2 & 0 & 2 & 0,7 & 2,7 \\
\hline Limoeiro & 1 & 0 & 1 & 0,3 & 1,8 \\
\hline $\begin{array}{l}\text { Nazaré da } \\
\text { Mata }\end{array}$ & 1 & 0 & 1 & 0,3 & 3,2 \\
\hline Paudalho & 1 & 0 & 1 & 0,3 & 1,9 \\
\hline Surubim & 1 & 0 & 1 & 0,3 & 1,7 \\
\hline $\begin{array}{l}\text { III Região de } \\
\text { saúde }\end{array}$ & 8 & 4 & 11 & 3,9 & ---- \\
\hline Água Preta & 2 & 0 & 2 & 0,7 & 6,0 \\
\hline Barreiros & 3 & 0 & 3 & 1 & 7,4 \\
\hline Gameleira & 0 & 1 & 1 & 0,3 & 3,6 \\
\hline $\begin{array}{l}\text { Lagoa dos } \\
\text { Gatos }\end{array}$ & 1 & 0 & 1 & 0,3 & 6,4 \\
\hline Palmares & 1 & 2 & 3 & 1 & 5,0 \\
\hline
\end{tabular}


Tabela 2. Continuação...

\begin{tabular}{|c|c|c|c|c|c|}
\hline \multirow[b]{2}{*}{$\begin{array}{l}\text { Região } \\
\text { de saúde/ } \\
\text { município }\end{array}$} & \multicolumn{5}{|c|}{ Tuberculose drogarresistente } \\
\hline & Secundária & Primária & Total & $\%$ & $\begin{array}{c}\text { Taxa de } \\
\text { incidência } \\
\text { (Total de } \\
\text { casos/ } \\
\text { habitantes } \\
\text { Censo } 2010 \mathrm{x} \\
100 \text { mil) }\end{array}$ \\
\hline Ribeirão & 0 & 1 & 1 & 0,3 & 2,3 \\
\hline Sirinhaém & 1 & 0 & 1 & 0,3 & 2,5 \\
\hline $\begin{array}{l}\text { IV Região de } \\
\text { saúde }\end{array}$ & 4 & 0 & 4 & 1,3 & ---- \\
\hline Agrestina & 1 & 0 & 1 & 0,3 & 4,4 \\
\hline Bonito & 1 & 0 & 1 & 0,3 & 2,7 \\
\hline Caruaru & 2 & 0 & 2 & 0,7 & 0,6 \\
\hline $\begin{array}{l}\text { V Região de } \\
\text { saúde }\end{array}$ & 3 & 0 & 3 & 1 & $x x$ \\
\hline Garanhuns & 3 & 0 & 3 & 1 & 2,3 \\
\hline $\begin{array}{l}\text { VI Região de } \\
\text { saúde }\end{array}$ & 1 & 0 & 1 & 0,3 & $x x$ \\
\hline Inajá & 1 & 0 & 1 & 0,3 & 5,2 \\
\hline $\begin{array}{l}\text { VII Região de } \\
\text { saúde }\end{array}$ & 1 & 0 & 1 & 0,3 & ---- \\
\hline Verdejante & 1 & 0 & 1 & 0,3 & 10,9 \\
\hline $\begin{array}{l}\text { VIII Região de } \\
\text { saúde }\end{array}$ & 5 & 2 & 7 & 2,3 & ---- \\
\hline Afrânio & 1 & 0 & 1 & 0,3 & 5,7 \\
\hline Petrolina & 3 & 2 & 5 & 1,7 & 1,7 \\
\hline $\begin{array}{l}\text { Santa Maria da } \\
\text { Boa Vista }\end{array}$ & 1 & 0 & 1 & 0,3 & 2,5 \\
\hline $\begin{array}{l}\text { IX Região de } \\
\text { saúde }\end{array}$ & 4 & 0 & 4 & 1,3 & ---- \\
\hline Araripina & 1 & 0 & 1 & 0,3 & 1,3 \\
\hline Ouricuri & 3 & 0 & 3 & 1 & 4,7 \\
\hline $\begin{array}{l}\text { XI Região de } \\
\text { saúde }\end{array}$ & 1 & 0 & 1 & 0,3 & ---- \\
\hline Serra Talhada & 1 & 0 & 1 & 0,3 & 1,3 \\
\hline $\begin{array}{l}\text { XII Região de } \\
\text { saúde }\end{array}$ & 1 & 0 & 1 & 0,3 & --- \\
\hline Goiana & 1 & 0 & 1 & 0,3 & 1,3 \\
\hline 40 municípios & 240 & 58 & 298 & 100 & ---- \\
\hline
\end{tabular}

A tendência temporal da taxa de incidência da TBDR em relação à população geral apresentou comportamento não linear, com dois momentos distintos: um entre 2002 e 2009, em que foi observado um comportamento estável, e outro momento ao longo dos anos de 2010 a 2014, com tendência ascendente (Figura 1).

No que se refere ao tipo da TBDR, dos 298 casos, 240 (80,5\%) foram de TBDR secundária, e 58 (19,5\%), de TBDR primária. O número absoluto de casos notificados, por ano, variou de 5 


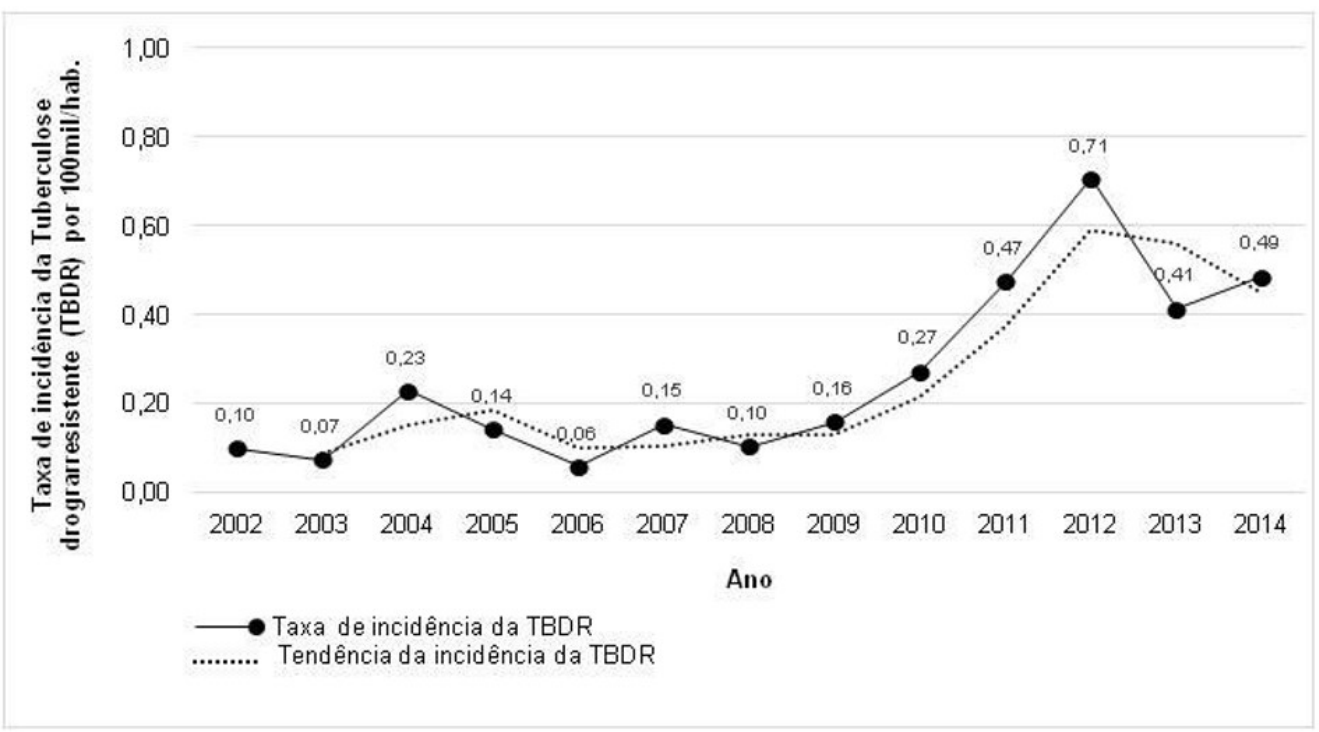

Nota: Incidência TBDR $=\underline{n^{\circ} \text { total de TBDR no ano }} \times 100.000$ habitantes

$\mathrm{n}^{\circ}$ total população geral no ano

Figura 1. Tendência da taxa de incidência da tuberculose drogarresistente (TBDR) em Pernambuco, 20022014. Incidência $T B D R=n^{\circ}$ total de TBDR no ano $\mathbf{x} \mathbf{1 0 0 . 0 0 0 ~ h a b i t a n t e s ~} / n^{\circ}$ total população geral no ano

a 63 casos, com uma média geral de 22 casos/ano. Nos cinco últimos anos da série histórica, houve a maior concentração de casos novos $(71,14 \% ; n=212)$. Em 2014, considerando em números absolutos, os casos de TBDR primária tiveram um crescimento de $333,3 \%$, e o de TBDR secundária, de 700\%, quando comparado ao início da série histórica (2002) (Figura 2).

A tendência temporal da proporção entre os casos de TBDR primária e secundária demonstrou comportamento não linear e dois momentos distintos. No período de 2002 a 2009, houve uma tendência decrescente, e a partir de 2010, a tendência apresentou-se crescente. Nos anos de 2006, 2008 e 2009, não houve casos de TBDR primária (Figura 3).

\section{DISCUSSÃO}

A TB-MDR e a TB-XDR são dois dos maiores desafios para os programas de controle da TB. Até o ano de 2050, cerca de 10 milhões de mortes estarão associadas à resistência antimicrobiana no mundo, sendo $25 \%$ em razão de cepas resistentes à TB ${ }^{19,20}$.

O referido estudo observou que os casos notificados de TBDR (298) em Pernambuco, no período de 2002 a 2014, corresponderam a 4,6\% dos casos do Brasil, colocando-o em 70 lugar entre os estados brasileiros com mais casos notificados no SITETB ${ }^{21}$.

ATB-MDR foi o padrão de resistência predominante na maioria dos casos de TBDR $(79,2 \%)$ de Pernambuco. $O$ tratamento prévio da TB tem sido descrito na literatura como um dos principais fatores de risco associado ao desenvolvimento da multirresistência ${ }^{22}$. Apesar da não avaliação dos fatores de riscos associados à TB-MDR, foi observado que a TBDR secundária foi frequente entre os casos de TB-MDR, ou seja, que já haviam realizado o tratamento para TB.

Concordando com outros estudos conduzidos no Brasil e em outros países, foi constatado que a TBDR acometeu, principalmente, pacientes do sexo masculino e adultos (de 25 a 49 anos) na faixa economicamente produtiva ${ }^{10,11,16,22-28}$.

Diferentemente deste estudo, a prevalência de TBDR foi semelhante entre os sexos masculino e feminino no Peru e no Paquistão ${ }^{29,30}$. As pesquisas realizadas em Henan (China) e em Lima (Peru) apontaram que a TBDR prevaleceu entre os maiores de 60 anos e na população de até 25 anos, respectivamente. A alta prevalência de idosos em Henan pode ter favorecido a 


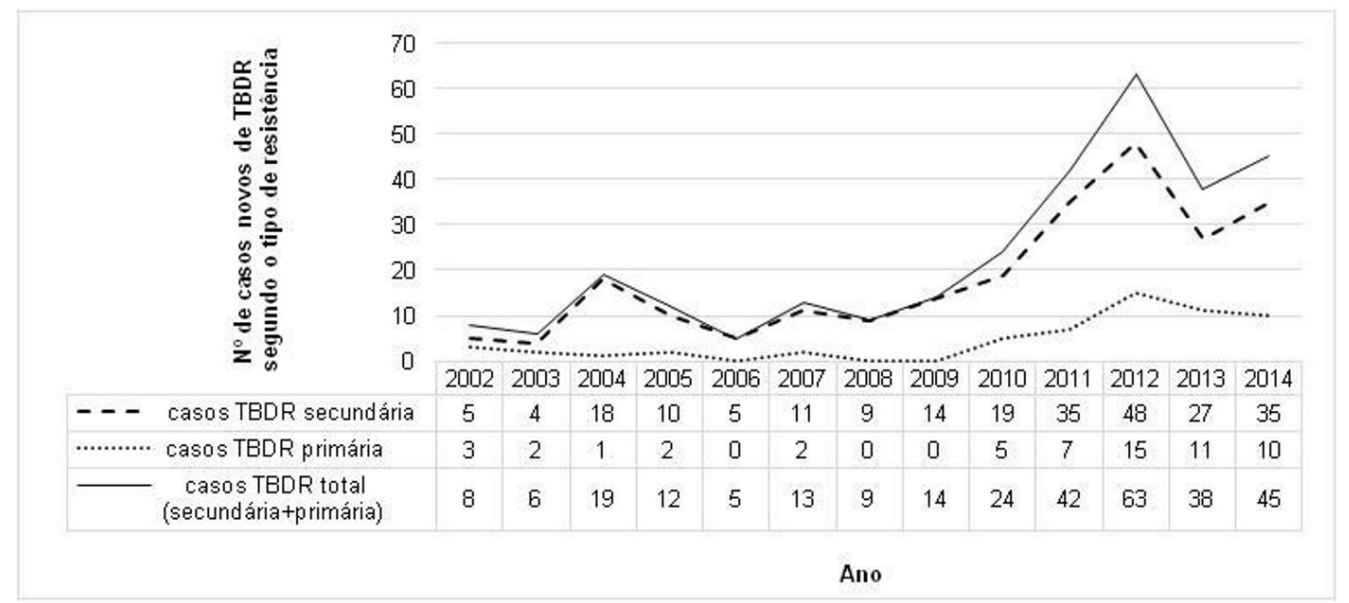

Figura 2. Número de casos novos de tuberculose drogarresistente (TBDR) segundo o tipo de resistência (primária e secundária) em Pernambuco, 2002-2014

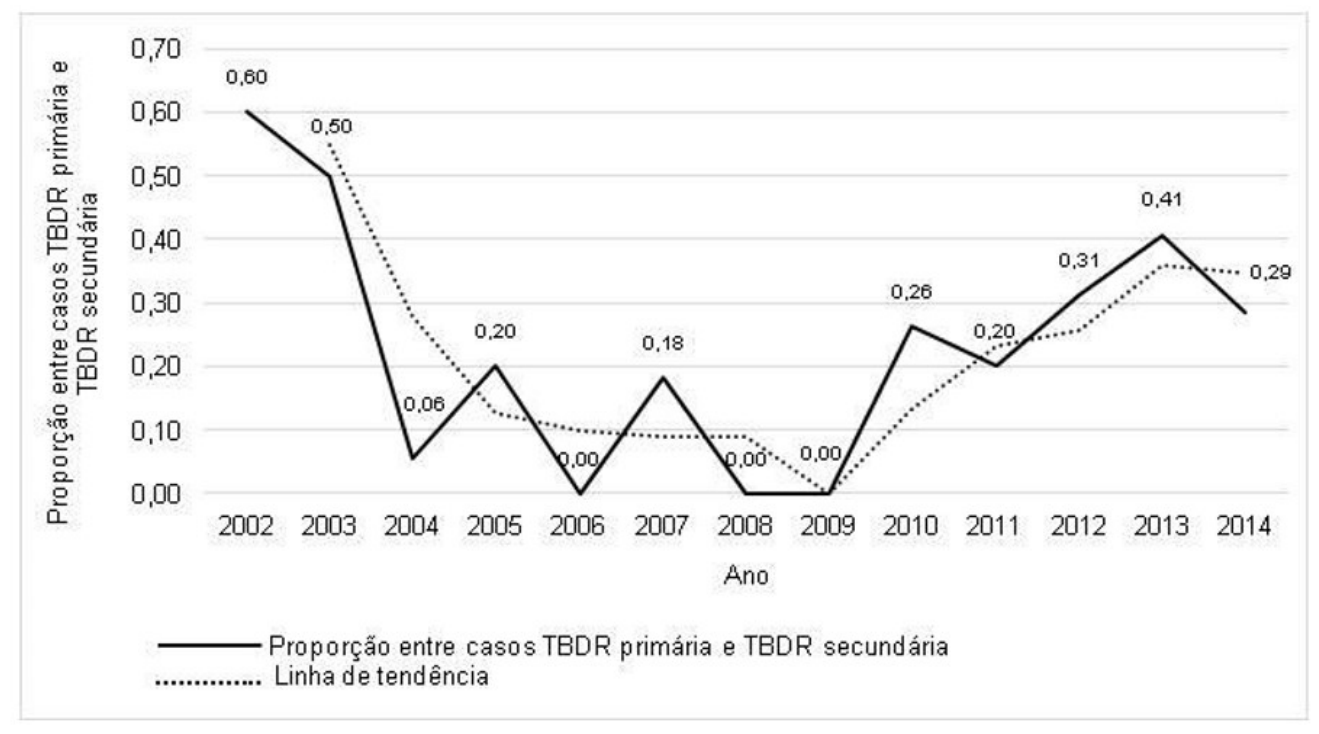

Nota: Proporção entre casos de TBDR primária e secundária $=\underline{n^{\circ} \text { total de TBDR primária no ano }}$ $\mathrm{n}^{\circ}$ total TBDR secundária no ano

Figura 3. Tendência da proporção entre casos de tuberculose drogarresistente (TBDR) primária e secundária em Pernambuco, 2002-2014. Proporção entre casos de TBDR primária e secundária $=n^{\circ}$ total de TBDR primária no ano / no total de TBDR secundária no ano

presença da TBDR nessa faixa etária, enquanto a alta taxa de TB em Lima pode ter contribuído para o maior risco do adoecimento por TBDR antes dos 25 anos $^{28,29}$.

A forma clínica pulmonar foi observada na maioria dos casos de TBDR deste estudo, o que pode ser presumível, uma vez que essa forma clínica é responsável pela transmissão direta da doença na população e é a mais comumente encontrada entre os casos de TB ${ }^{23,24,31}$.

A realização do TDO é uma importante estratégia para o controle da TB e TBDR, sendo relevante para a obtenção de desfechos favoráveis (cura e tratamento completo) e prevenção do desenvolvimento de casos de TB-MDR e TB-XDR ${ }^{24}$.

Neste estudo, foi constatado que a maioria dos pacientes $(61,7 \%)$ com TBDR da série histórica não tiveram um TDO, sendo esse percentual acima do que é recomendado pelo 
Programa Nacional do Controle da Tuberculose (PNCT) do Brasil ${ }^{12}$. A frequência da realização de TDO nos casos de TBDR variou entre os estados brasileiros. Paro Pedro et al. (2015), no estado de São Paulo, identificaram que $48,4 \%$ dos casos de TBDR foram acompanhados por TDO. Nos estados do Paraná $(74,4 \%)$ e do Tocantins $(68,1 \%)$, as coberturas de realização do TDO foram as mais altas do Brasil. No estado do Rio Grande do Sul, essa cobertura foi apenas de $17,2 \%$, sendo considerada a mais baixa entre os estados brasileiros. Provavelmente esses dados estejam relacionados às políticas de saúde de cada região, do comprometimento das equipes de saúde e da quantidade de recursos humanos disponíveis para a realização do TDO ${ }^{14}$.

Neste estudo, $41,6 \%$ dos casos apresentaram desfecho do tratamento não favorável, entre os quais 1/3 foi a óbito por TBDR. Corroborando os achados desta pesquisa, vários estudos mostram que, no contexto geral, o indicador de cura entre os indivíduos com TBDR estão abaixo do recomendado pela Organização Mundial da Saúde (OMS) e que os percentuais de abandono e óbitos ainda são elevados ${ }^{3,10,22,32,33}$. Portanto, o desfecho não favorável contribui para a perpetuação da cadeia de transmissão da doença.

A notificação do número de casos de TBDR neste estudo variou entre as cidades de Pernambuco, havendo uma concentração nos municípios da Região Metropolitana de Recife, localizados na I GERES. Apesar de Recife ter notificado o maior número de casos de TBDR da série histórica, esse município não esteve entre os cinco municípios do estado que apresentaram as maiores chances de ocorrência da TBDR. Tais fatos podem estar relacionados à existência de heterogeneidade territorial, social e econômica, bem como facilidade de acesso dos pacientes aos serviços de saúde e hospitais terciários de referência, localizados em Recife, capital do estado de Pernambuco ${ }^{34,35}$.

Durante o período de 2002 a 2014, a incidência da TBDR em Pernambuco não apresentou valores anuais de incidências maiores que $1,0 / 100$ mil habitantes, ficando abaixo do que a OMS estimou em 2013 (6,6/100 mil habitantes), levando em consideração que, provavelmente, a maioria desses casos tenha sido subdiagnosticado. Países como Canadá, Estados Unidos, Porto Rico, Uganda, África do Sul, Rússia, China, Vietnã, Coreia do Sul, Índia, Bolívia, Colômbia, Peru e alguns países da Europa também apresentaram resultados de incidência semelhantes ao deste estudo ${ }^{36,37,38}$. Entre a população de alguns desses países, Rockwood et al. (2015), em um estudo de metanálise, observaram uma incidência média cumulativa da TBDR de 0,1 (IC95\%, 0,07-3,2\%), considerando-a como um evento raro ${ }^{33,36}$. Diferindo dos resultados encontrados para Pernambuco, a taxa de incidência da TBDR observada por Coelho et al. (2012), em Santos, São Paulo (SP), Brasil, apresentou uma média anual de 1,9/100 mil habitantes ${ }^{10}$.

Expressivamente, houve uma heterogeneidade das incidências encontradas na série histórica (2002-2014), o que dificultou a interpretação de como a tendência da TBDR em Pernambuco poderia se comportar nos próximos anos. A instabilidade na incidência encontrada neste estudo pode estar associada a fragilidades do programa de controle da TB no estado, como: dificuldade de consolidação das atividades do programa pelos profissionais de saúde, em especial no nível primário de atenção e vigilância; dificuldade de acesso ao diagnóstico da TBDR; limitado conhecimento dos profissionais acerca do diagnóstico e tratamento da TBDR; falha de acesso dos casos resistentes aos serviços terciários de referência para notificação e acompanhamento adequado.

Neste estudo, foi observado aumento na incidência de casos novos de TBDR nos últimos cinco anos (2010-2014), o que pode estar relacionado à efetivação das ações promovidas para o enfrentamento da TB no estado de Pernambuco, a partir da implantação do Programa SANAR, em 2011, que, entre as ações prioritárias, proporcionou: capacitação técnica para os profissionais da assistência e vigilância, descentralização e ampliação dos exames diagnósticos da TB para todas as GERES ${ }^{39}$. Outro fator que é importante destacar e que pode ter contribuído para o aumento da notificação dos casos da TBDR nos últimos anos da série histórica foi a implantação e a capacitação do uso do sistema informatizado, o SITETB, no período entre 2011 e $2013^{12,13}$.

Corroborando outros estudos, foi constatado que a TBDR secundária foi predominante em relação à TBDR primária ${ }^{10,22,40,41}$. O abandono do tratamento da TB sensível tem sido considerado uma das principais causas associadas ao desenvolvimento da TBDR secundária 6,21,26,42,43,44. 
Apesar da escassez na literatura de estudos em relação à tendência das proporções entre a TBDR primária e secundária, essa variável pode ser um indicador sensível para avaliar o desempenho do PNCT, sobretudo quando existe o predomínio da TBDR primária, demonstrando o risco aumentado da disseminação de cepas resistentes na comunidade ${ }^{10}$.

A tendência das proporções da TBDR primária e secundária encontrada ao longo da série histórica não se apresentou linear, indicando variações instáveis no período do estudo, dificultando a previsão dessa relação (primária e secundária) para os anos seguintes. Ademais, este estudo possui limitações metodológicas, e uma delas é referente ao próprio tipo de estudo. Por se tratar de banco de dados secundários e de uma análise retrospectiva, alguns dados podem estar subestimados, não representando a totalidade dos aspectos relacionados ao perfil epidemiológico e aos dados de incidência da TBDR em Pernambuco. Mesmo com essa limitação, os resultados observados neste estudo permitiram estimar a incidência, o perfil sociodemográfico e clínico-epidemiológico em relação à TBDR no estado. Essas informações podem subsidiar profissionais de saúde e gestores locais na identificação da população mais atingida, a fim de estabelecer melhores estratégias para o controle da doença no âmbito local, em especial para a detecção e a cura desses casos.

Até o momento, esta pesquisa, provavelmente, deva ser a primeira análise sobre o estudo de tendência temporal da TBDR em Pernambuco. Apesar de, na série histórica estudada, a TBDR apresentar baixas taxas de incidência e a tendência temporal não ter comportamento linear, foi observada uma curva crescente da incidência da doença nos últimos anos (20102014) estudados (Figura 1), podendo ser considerado um sinal de alerta, demonstrando que a resistência é um problema evidente de saúde pública e um desafio para os programas de controle da TB em Pernambuco.

Concluindo, pela escassez de dados nessa área, os resultados deste estudo geraram informações científicas relevantes, de acordo com a realidade das regiões do Nordeste do Brasil em relação à TBDR, possibilitando, assim, subsidiar a melhoria das políticas públicas de saúde e a qualidade de vida da população e reforçar as ações de vigilância no sentido de identificar o maior número de casos portadores da doença, contribuindo para os gestores aprimorarem os programas de controle da TBDR.

\section{AGRADECIMENTOS}

À Secretaria Estadual de Saúde de Pernambuco, pela disponibilização da base de dados.

\section{REFERÊNCIAS}

1. World Health Organization. Global tuberculosis report 2017. Geneva: World Health Organization; 2017.

2. World Health Organization. Global tuberculosis report 2014. Geneva: World Health Organization; 2014.

3. World Health Organization. Global tuberculosis report 2016. Geneva: World Health Organization; 2016.

4. Câmara JT, Menezes JB, Pereira BM, Oliveira TRC, Oliveira TMP, Ribeiro NS. Perfil epidemiológico de pacientes com diagnóstico de resistência de Mycobacterium tuberculosis. Rev enferm UFPE on line. 2016;10(11):4082-9.

5. Coelho AGV.Tuberculose multirresistente e extensivamente resistente em área metropolitana de elevada incidência [tese]. Santos (SP): Faculdade de Medicina, Universidade de São Paulo; 2014.

6. Arakaki-Sanchez D, Brito RC. Manual de recomendações para o controle da tuberculose no Brasil. Brasília: Ministério da Saúde, 2011. 284 p.

7. Matteelli A, Roggi A, Carvalho ACC. Extensively drug-resistant tuberculosis: epidemiology and management. Clin Epidemiol. 2014;6:111-8. http://dx.doi.org/10.2147/CLEP.S35839 PMid:24729727.

8. World Health Organization. Global tuberculosis control: WHO report 2011. Geneva: World Health Organization; 2011.

9. World Health Organization. Global tuberculosis report 2013. Geneva: World Health Organization; 2013.

10. Coelho AGV, Zamarioli LA, Telles MA, Ferrazoli L, Waldman EA. A study of multidrug-resistant tuberculosis in risk groups in the city of Santos, São Paulo, Brazil. Mem Inst Oswaldo Cruz. 2012;107(6):760-6. http:// dx.doi.org/10.1590/S0074-02762012000600009 PMid:22990966. 
11. Mulisa G, Workneh T, Hordofa N, Suaudi M, Abebe G, Jarso G. Multidrug-resistant Mycobacterium tuberculosis and associated risk factors in Oromia Region of Ethiopia. Int J Infect Dis. 2015;39:57-61. http://dx.doi.org/10.1016/j.jiid.2015.08.013 PMid:26327121.

12. Boletim epidemiológico. Brasília: Secretaria de Vigilância em Saúde. Vol. 46, No. 9, 2015.

13. Boletim epidemiológico. Recife: Secretaria de Saúde. Vol. 3, No. 4, 2014.

14. Boletim epidemiológico. Brasília: Secretaria de Vigilância em Saúde. Vol. 48, No. 8, 2017.

15. Boletim epidemiológico. Brasília: Secretaria de Vigilância em Saúde. Vol. 44, No. 02, 2014.

16. Vieira RCA, Fregona G, Palaci M, Dietze R, Maciel ELN. Perfil epidemiológico dos casos de tuberculose multirresistente do Espírito Santo. Rev Bras Epidemiol. 2007;10(1):56-65. http://dx.doi.org/10.1590/S1415$790 \times 2007000100007$.

17. Secretaria Estadual de Saúde de Pernambuco. Plano Estadual de Saúde 2012-2015. 1. ed. Recife: Secretaria Estadual de Saúde; 2012. 247 p.

18. Box GEP, Jenkins GM. Time series analysis: forecasting and control. Oakland: HoldenDay; 1976.

19. O'Neill, J. Tackling drug-resistant infections globally: final report and recommendations [Internet]. London: Government of the United Kingdom; 2016 [citado em 2016 maio 13 ]. (Review on Antimicrobial Resistance). Disponível em: https://apo.org.au/node/63983.

20. World Health Organization. The end TB strategy. Genebra: World Health Organization; 2016.

21. Boletim epidemiológico. Brasília: Secretaria de Vigilância em Saúde. Vol. 47, No. 13, 2016.

22. Lomtadze N, Aspindzelashvili R, Janjgava M, Mirtskhulava V, Wright A, Blumberg HM, et al. Prevalence and risk factors for multidrug-resistant tuberculosis in Republic of Georgia: a population based study. Int J Tuberc Lung Dis. 2009;13(1):68-73. PMid:19105881.

23. Ferreira KR, Cavalcante EGR, De-La-Torre-Ugarte-Guanilo MC, Berti RAL, Bertolozzi MR. Portadores de tuberculose multirresistente em um centro de referência: perfil sócio-demográfico e clínicoepidemiológico. Rev Esc Enferm USP. 2011;45(2 N. esp.):1685-9. http://dx.doi.org/10.1590/S008062342011000800008 PMid:22569655.</jrn>

24. Pedro HSP, Nardi SMT, Pereira MIF, Oliveira RS, Suffys PN, Gomes HM, et al. Clinical and epidemiological profiles of individuals with drug-resistant tuberculosis. Mem Inst Oswaldo Cruz. 2015;110(2):235-41. http://dx.doi.org/10.1590/0074-02760140316 PMid:25946248.

25. Shen X, DeRiemer K, Yuan ZA, Shen M, Xia Z, Gui X, et al. Drug resistant tuberculosis in Shanghai, China, 2000-2006: prevalence, trends, and risk factors. Int J Tuberc Lung Dis. 2009;13(2):253-9. PMid:19146756.

26. Micheletti VCD, Moreira JS, Ribeiro MO, Kritski AL, Braga JU. Drug-resistant tuberculosis in subjects included in the Second National Survey on Antituberculosis Drug Resistance in Porto Alegre, Brazil. J Bras Pneumol. 2014;40(2):155-63. http://dx.doi.org/10.1590/S1806-37132014000200009 PMid:24831400.

27. Rifat $M$, Milton $A H$, Hall J, Oldmeadow $C$, Islam MA, Husain A, et al. Development of multidrug resistant tuberculosis in Bangladesh: a case-control study on risk factors. PLoS One. 2014;9(8):e105214. http:// dx.doi.org/10.1371/journal.pone.0105214 PMid:25136966.

28. Zhang C, Wang Y, Shi G, Han W, Zhao H, Zhang H, et al. Determinants of multidrug-resistant tuberculosis in Henan province in China: a case control study. BMC Public Health. 2015;16(1):42. http://dx.doi.org/10.1186/ s12889-016-2711-z PMid:26775263.

29. Otero L, Krapp F, Tomatis C, Zamudio C, Matthys F, Gotuzzo E, et al. High Prevalence of primary multidrug resistant tuberculosis in persons with no known risk factors. PLoS One. 2011;6(10):e26276. http://dx.doi. org/10.1371/journal.pone.0026276 PMid:22046266.

30. Ullah I, Javaid A, Tahir Z, Ullah O, Shah AA, Hasan F, et al. Pattern of drug resistance and risk factors associated with development of drug resistant mycobacterium tuberculosis in Pakistan. PLoS One. 2016;11(1):e0147529. http://dx.doi.org/10.1371/journal.pone.0147529. PMid:26809127.

31. Viana PVS, Redner P, Ramos JP. Fatores associados ao abandono e ao óbito de casos de tuberculose drogarresistente (TBDR) atendidos em um centro de referência no Rio de Janeiro, Brasil. Cad Saude Publica. 2018;34(5):e00048217. http://dx.doi.org/10.1590/0102-311x00048217. PMid:29768580.

32. Orenstein EW, Basu S, Shah NS, Andrews JR, Friedland GH, Moll AP, et al. Treatment outcomes among patients with multidrug- resistant tuberculosis: systematic review and meta-analysis. Lancet Infect Dis. 2009;9(3):153-61. http://dx.doi.org/10.1016/S1473-3099(09)70041-6. PMid:19246019.

33. Moyo S, Cox HS, Hughes J, Daniels J, Synman L, De Azevedo V, et al. Loss from treatment for drug resistant tuberculosis: risk factors and patient outcomes in a community-based program in Khayelitsha, South Africa. PLoS One. 2015;10(3):e0118919. http://dx.doi.org/10.1371/journal.pone.0118919. PMid:25785451. 
34. Maciel LEL, Reis-Santos B. Determinants of tuberculosis in Brazil: from the conceptual framework to the practical application. Rev Panam Salud Publica. 2015;38(1):28-34. PMid:26506318.

35. Pinheiro RS, de Oliveira GP, Oliveira EX, Melo EC, Coeli CM, Carvalho MS. Determinantes sociais e autorrelato de tuberculose nas regiões metropolitanas conforme a Pesquisa Nacional por Amostra de Domicílios, Brasil. Rev Panam Salud Publica. 2013;34(6):446-51. PMid:24569974.

36. Rockwood N, Abdullahi LH, Wilkinson RJ, Meintjes G. Risk Factors for Acquired rifamycin and isoniazid resistance: a systematic review and meta- analysis. PLoS One. 2015;10(9):e0139017. http://dx.doi. org/10.1371/journal.pone.0139017 PMid:26406228.

37. Cohen T, Jenkins HE, Lu C, McLaughlin M, Floyd K, Zignol M. On the spread and control of MDR-TB epidemics: an examination of trends in anti-tuberculosis drug resistance surveillance data. Drug resist updat. 2014;17(4-6):105-23. http://dx.doi.org/10.1016/j.drup.2014.10.001 PMid:25458783.

38. United Nations. The millennium development goals report 2015. New York: United Nations Publications; 2015.

39. Secretaria Estadual de Saúde de Pernambuco. Plano Integrado de Ações para o Enfrentamento às Doenças Negligenciadas no Estado de Pernambuco/SANAR - 2015-2018. Recife: Secretaria Estadual de Saúde; 2015. 46 p.

40. Rocha JL, Dalcolmo MP, Federle D, Marques, MG. Tuberculose multirresistente. Pulmão RJ. 2008;17(1):2732.

41. Marques M, Cunha EA, Ruffino-Netto A, Andrade SM. Perfil de resistência de Mycobacterium tuberculosis no estado de Mato Grosso do Sul, 2000-2006. J Bras Pneumol. 2010;36(2):224-31. http://dx.doi.org/10.1590/ S1806-37132010000200011. PMid:20485944.

42. World Health Organization. Anti-tuberculosis drug resistance in the world: fourth global report. Geneva: World Health Organization; 2008.

43. Kritski AL. Emergência de tuberculose resistente: renovado desafio. J Bras Pneumol. 2010;36(2):157-8. http://dx.doi.org/10.1590/S1806-37132010000200001. PMid:20485934.

44. Chien JY, Lai CC, Tan CK, Chien ST, Yu CJ, Hsueh PR. Decline in rates of acquired multidrug-resistant tuberculosis after implementation of the directly observed therapy, short course (DOTS) and DOTS-Plus programmes in Taiwan. J Antimicrob Chemother. 2013;68(8):1910-6. http://dx.doi.org/10.1093/jac/dkt103. PMid:23580558. 